\title{
The detection of phase shifts in two-dimensional images
}

\author{
TERRY CAELLI, MARTIN HÜBNER, and INGO RENTSCHLER \\ Institute for Medical Psychology, University of Munich, Federal Republic of Germany
}

\begin{abstract}
The ability of observers to register the spatial luminance gradients of images in specific locations can be loosely defined by their sensitivity to spatial phase. Here we have investigated the detectability of phase shifts in two-dimensional images as a function of their center frequencies and bandwidths. In three separate experiments, involving the discrimination of such signals in terms of the addition of constant phase offsets to each target frequency component, we have established that: (1) sensitivity to phase shifts is largely independent of a signal's center frequency; (2) sensitivity increases with increases in the phase-shift bandwidth and is extremely weak below 1-octave-wide signals; and (3) sensitivity markedly improves with the addition of a reference frequency band to the two signals but decreases with the spectral distance between the reference and phase-shifted regions.
\end{abstract}

As recently demonstrated by Oppenheim and Lim (1981) and Brettel, Caelli, Hilz, and Rentschler (1982), the Fourier amplitude and phase spectra of an image depict quite different aspects of the spatial-frequency components that compose an image. While amplitudes determine the contrast of each component grating, the phase values define each component's relative position with respect to the image coordinate system. Image fidelity is markedly affected when such positional information is altered, since the phase spectrum encodes the spatial arrangement of frequency components defining the spatial pattern or image structure. Caelli and Bevan (1982) and Caelli and Hübner $(1983,1984)$ have recently demonstrated that human observers are more sensitive to information reductions in phase than in amplitude. That is, in order to preserve image quality, image phase spectra can rarely be resolved into phase angles broader than $45^{\circ}$ apart and, in some cases, phase resolutions of up to $15^{\circ}$ are required (Caelli $\&$ Bevan, 1982). Out of the full $360^{\circ}$ permissible phase range, such resolutions demand between 3 and 5 bits of information whereas image power (amplitude) can be stored in considerably fewer bits (Caelli \& Hübner, 1983, 1984).

Perhaps the strongest demonstration of the importance of spatial phase (compared with power) in image coding is that images reconstructed by setting the amplitude to a constant, though unaltered in phase, appear as highfrequency emphasized versions of the input image. However, images reconstructed by setting the phase components to a constant, though unaltered in amplitude, appear as noise (Oppenheim \& Lim, 1981). Such results run counter to extant claims in the literature (for example,

\footnotetext{
This project was funded by grants from the Deutsche Forschungsgemeinschaft $(\operatorname{Re} 337 / 4-1)$ and the Natural Sciences and Engineering Research Council of Canada (A2568). Reprint requests should be directed to T. M. Caelli, Psychology Department, The University of Alberta, Edmonton, Alberta, Canada T6G 2E9.
}

Atkinson \& Campbell, 1974; Burr, 1979) that phase sensitivity, relative to that for amplitude, is low.

Sensitivity to spatial phase can be viewed as a generalization of position or vernier acuity when the ability of observers to locate positional information is studied as a function of spatial frequency (Westheimer, 1978). Results with one-dimensional gratings are conflicting, although resolutions between phase angles smaller than $15^{\circ}$ have not been reported (Atkinson \& Campbell, 1974; Burr, 1979; Gayl, Strasburger, Rentschler, \& Hilz, 1982; Nachmias \& Weber, 1975). Employing the operation of "phase quantization," in which image phase spectra values were resolved to a limited number of states (bits), Caelli and Bevan (1982) observed the ability of observers to detect depletions in such phase information in two-dimensional textures. Results showed perceptual resolutions (the inability of observers to discriminate original from phase quantized images) to be no better than $15^{\circ}$ and in many cases as low as $45^{\circ}$, depending on the spectral energy distribution of the suprathreshold images employed. In addition, no evidence, once spectral energy (or amplitude) differences are considered, has been found for variations in sensitivity over different spatial frequency bands (Caelli \& Bevan, 1982).

The experiments to be reported here made use of a more direct way to determine phase sensitivity in twodimensional visual image processing-employing the shifting of specified phase spectra components. Such a process is readily interpreted in the image (retinotopic) domain (Burton \& Moorhead, 1981) in terms of the "decorrelation' of specific luminance gradients (frequency components) from others.

Specifically, phase shifting refers to the addition of a constant phase value $(\Delta \phi)$ to all phase states of a given spatial-frequency region of the image spectrum shown in Figure la. By the shifting theorem (Caelli, 1981), it is clear that this operation can be interpreted in the following way. Each point, or more precisely, each conjugate 
pair of points, in the band-limited region of Figure 1a corresponds to an oriented $\left[\theta=\tan ^{-1}(\mathrm{v} / \mathrm{u})\right]$ grating of spatial frequency $f=\left(u^{2}+v^{2}\right)^{1 / 2}$, with amplitude $A(u, v)$ and phase $\phi(u, v)$ defined by

$$
A(u, v)=\left[R^{2}(u, v)+I^{2}(u, v)\right]^{1 / 2}
$$

and

$$
\phi(\mathrm{u}, \mathrm{v})=\tan ^{-1}[\mathrm{I}(\mathrm{u}, \mathrm{v}) / \mathrm{R}(\mathrm{u}, \mathrm{v})] .
$$

Here, $R(u, v)$ and $I(u, v)$ correspond to the real and imaginary spectral components at the spectral coordinates $(u, v)$ measured in cycles per degree (cpd) of visual angle, thus defining a grating in the image domain. A phase shift of $\Delta \phi$ has the effect of translating the grating by $\Delta \phi$, or $\Delta \phi / 2 \pi \mathrm{f}$ degrees of visual angle, in a direction parallel to the grating's modulation axis $(\theta$, see above). For the bandlimited region in Figure 1a, this creates displacements of between $r_{\ell}=\Delta \phi / 2 \pi f_{u}$ and $r_{u}=\Delta \phi / 2 \pi f_{p}$ in Figure 1b. Furthermore, since all orientations are involved, the net effect is to create a "shifting window" (an annulus in this case) about every point in the image domain defined by the phase-shift value $\Delta \phi$ and the frequency band limits $f_{\ell}$ (lower frequency cutoff) and $f_{u}$ (upper frequency cutoff), as shown in Figure 1. This window is analogous to a transfer function that determines the modulation of amplitude in a linear system. That is, loss of amplitude is measured by a modulating factor, whereas loss of phase is represented by the addition/subtraction of phase angles (position). (For an analogy of such phase-shifting operations in physical optics, see the Discussion section.)

Consider two identical images. A phase shift of $\Delta \phi$ is introduced to one of these images in a frequency band, as defined in Figure 1a. The two images will then be spatially decorrelated, and the extent of the decorrelation is defined by the geometry of the shifting window, the spectral density, and the energy of the bandwidth (Figure 1). Since both images would have identical spectral densities, discrimination could not occur in terms of differences between energy states in a set of spatial-frequency channels. Rather, the detection of such a decorrelation is more likely to be related to a correlation process, whereby the observer would perceptually cross-correlate the two images in order to discriminate between them as a function of the phase shift.

In the following experiments, we attempted to answer a number of questions regarding the sensitivity of human observers to luminance decorrelations created by such phase shifts, though no direct attempt is made in this report to assess the proposed cross-correlation mechanism (see Caelli \& Rentschler, 1985).

\section{EXPERIMENT 1: \\ Phase-Shift Detection as a Function of Frequency Bandwidth}

Of particular importance for the detection of phaseshifting effects is the bandwidth of the phase-modulated region, as illustrated in Figure 1. Studies with onedimensional grating patterns (e.g., Nachmias \& Weber, 1975 ) suggest that phase "encoding" is accomplished via broadly tuned spatial-frequency channels. If generalized to the suprathreshold two-dimensional image domain, this claim would predict that sensitivity to phase-shift-induced luminance profile differences would be more acute with broad-bandwidth shifts. In the following experiment, we studied this prediction at two center frequencies with random checkerboard patterns by bandpass filtering the images in different bandwidths before phase-shifting one of a pair of initially identical images.

\section{Method}

Apparatus and Stimuli. All images were generated as $128 \times$ 128 6-bit (64 gray level, linear with luminance in the chosen range) pixel random checkerboard textures. They were displayed by a Videograph image processing system and a Grundig television monitor with $\mathrm{P} 32$ phosphor. All images subtended a fixed $2^{\circ}$ visual angle to the observer at a distance of $2.14 \mathrm{~m}$ from the screen.

Two center frequencies (Figure 1) were chosen at 6 and $12 \mathrm{cpd}$, each having associated phase-shifting bandwidths of $1,2,3$, and 4 octaves' total width. Phase shifts of $0^{\circ}$ (original) to $90^{\circ}\left(10^{\circ}\right.$, $20^{\circ}, 30^{\circ}, 40^{\circ}, 50^{\circ}$ for 3 and 4 octaves; $50^{\circ}, 60^{\circ}, 70^{\circ}, 80^{\circ}, 90^{\circ}$ for the 2-octave range) were used with the eight filter ranges, resulting in a basic $8 \times 6=48$ images. No observers could detect (discriminate) any of the phase shifts (including ones greater than $90^{\circ}$ ) with the 1-octave-wide signals, and those results have not been included (Figure 2). Amplitude values were set to zero (bandpass filter) outside each region, but were untouched within. Inverse FFTs were then computed to result in each of the 48 images' having a constant fixed space average luminance of $60 \mathrm{~cd} / \mathrm{m}^{2}$. All images spanned the complete 6-bit intensity levels with identical peak contrast values of $0.8\left[\left(\ell_{\max }-\ell_{\min }\right) /\left(\ell_{\max }+\ell_{\min }\right), \ell_{\max }, \ell_{\min }\right.$ corresponding to maximum and minimum luminances]. Examples are shown in Figure 2.

Subjects and Procedure. Two observers were used, one with uncorrected normal acuity (WE) and the other with corrected nor mal acuity (MH). A signal-detection rating-scale procedure, as described by Green and Swets (1974, pp. 40-43), was employed. A given trial consisted of the sequential presentation of two images (in the identical spatial area), which the observer was required to judge as being the same or different. The observer's responses were made using confidence ratings from 1 to 3 for same ( $1=$ very certain) and from 4 to 6 for different $(6=$ very certain $)$. (a)

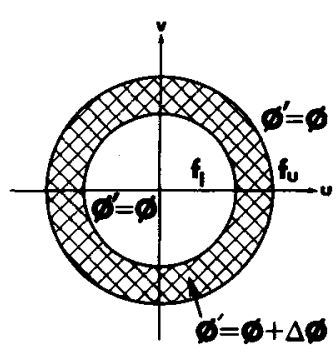

(b)

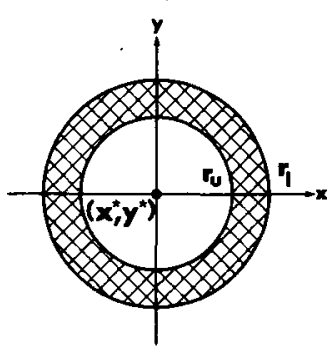

Figure 1. (a) Spectral definition of two-dimensional phase-shifting operation $\left[\phi^{\prime}=g(\phi)\right]$, and (b) the associated shifting or "shifting window" occurring at every position $\left(\mathrm{x}^{*}, \mathrm{y}^{*}\right)$ in the image domain. Here $u, v$ refer to spectral coordinates in cycles per degree and $x, y$ refer to retinotopic coordinates in degrees of visual angle. $r_{q}, r_{u}$ correspond to $\Delta \phi / 2 \pi f_{u}, \Delta \phi / 2 \pi f_{l}$, respectively, for $\Delta \phi=$ phase shift and $f=\sqrt{u^{2}+v^{2}}$. This shifting window is analogous to the transfer function of a filter defined in the amplitude domain. 

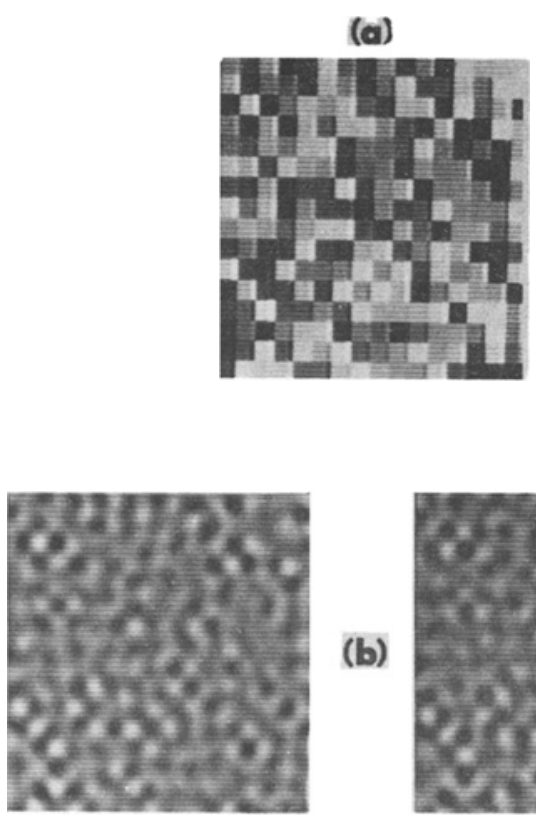

(b)
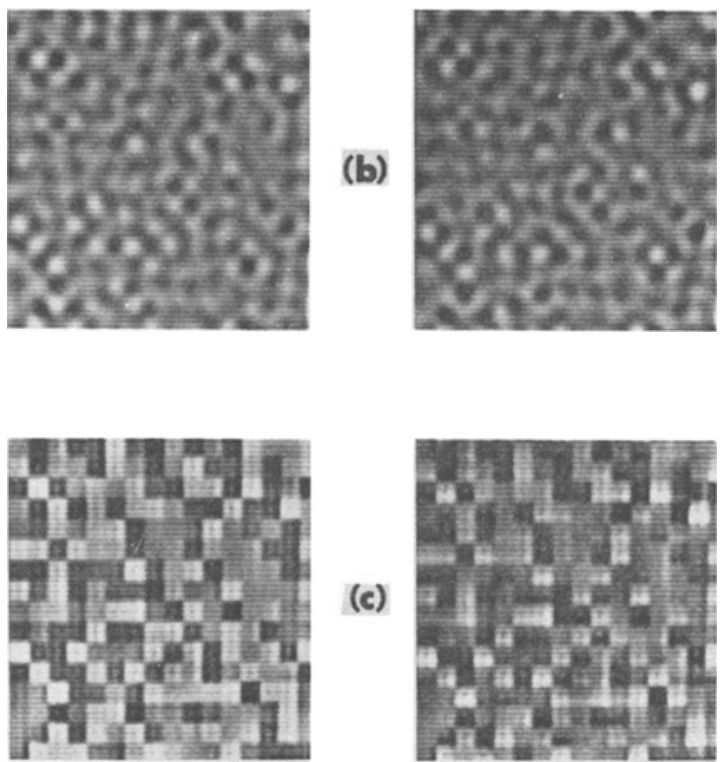

(c)

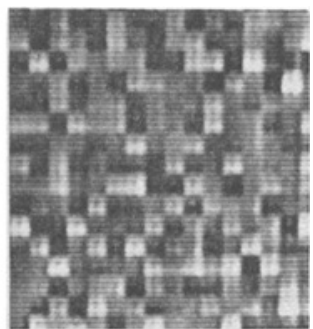

Figure 2. (a) Original random checkerbaard texture $(128 \times 1286-$ bit pixel). (b) Two 1-octave ( $4<\mathrm{f} \leq 8 \mathrm{cpd}$ in this experiment) bandpass versions, with $\Delta \phi=0^{\circ}$ and $90^{\circ}$, respectively. (c) Equivalent 3-octave $(2<\mathrm{f} \leq 16 \mathrm{cpd})$ versions with $\Delta \phi=0^{\circ}$ and $50^{\circ}$. In the latter case, discrimination is possible.

An experimental run consisted of 20 replications of each $\Delta \phi$ for a given phase-shift range and 40 catch trials (with zero phase shift), resulting in 140 trials per condition. Each observer repeated this sequence three times, giving a total of $140 \times 3 \times 8=3,360$ trials. Stimuli were allocated randomly over trials, with the exposure time set at $200 \mathrm{msec} / \mathrm{signal}$ and an interstimulus interval of $700 \mathrm{msec}$. The observers were instructed to fixate the center of a viewing aperture at all times. Sequential displays were used so that the images covered exactly the same fixation area-given the brief exposure times employed. Exposure times were limited to $200 \mathrm{msec}$ to assure fixation and limited eye movements. However, an adequate interstimulus interval $(700 \mathrm{msec})$ was required to avoid possible masking effects.

\section{Results and Discussion}

In accordance with the method outlined by Green and Swets (1974), discrimination sensitivity was measured using $P(A)$, the area under the receiver-operating characteristic (ROC) curve. This method transforms hit and false-alarm probabilities into detectability or discriminability $[\mathrm{P}(\mathrm{A})]$ scores, which vary between 0.5 and 1.0 , where 0.75 is equivalent to the $75 \%$ detection level ob- tained from a two-alternative forced-choice procedure and 0.5 corresponds to guessing (Dorfman \& Alf, 1969; Green \& Swets, 1974, pp. 45-50; Harvey, 1984).

Figure 3 shows $\mathrm{P}(\mathrm{A})$ results for the two observers over the experimental conditions. These data show an improvement in phase-shift sensitivity with increases in frequency bandwidth-particularly when the bandwidth is greater than 1 octave about the center frequencies, since no observer could detect the shift with 1-octave-wide shifts. This increase in sensitivity decreases in size with larger bandwidths (compare the difference between 2 and 3 octaves with that between 3 and 4 octaves).

These data demonstrate that the perceptual sensitivity to phase-shift decorrelations in the image luminance profiles is near maximum at about a 2-octave signal bandwidth, with only slight improvement for wider ranges. It is also important to note that the results are almost identical for the two frequency ranges. This suggests that the detection of such decorrelations is dependent not on absolute shift values but rather on the relative decorrelation of luminance information within any given bandwidth region. Given these conclusions, then, we would expect that the detection of phase shifts within a relatively narrow band would be enhanced if correlated information was added to both images, particularly if it was spectrally near the decorrelated signals. This hypothesis was investigated in Experiment 2.

EXPERIMENT 2:

\section{Phase-Shift Sensitivity in the Presence of Correlated Band-Limited Information}

\begin{abstract}
Method
Apparatus and Procedure. This experiment was identical to the previous one except that a fixed low-frequency (correlated) band was added to the original and phase-shifted (higher) bandpass images. This lower bandpass region was fixed at $f \leq 4 \mathrm{cpd}$ and was added to each image with identical amplitude and phase values. The higher frequency regions were varied from $4<\mathrm{f} \leq 8 \mathrm{cpd}$ to $6<\mathrm{f} \leq 12 \mathrm{cpd}$ and $8<\mathrm{f} \leq 16 \mathrm{cpd}$. As before, phase shifts were introduced in one image but not in the other within each band. One-octave bandwidths were chosen here because we had previously found that such bandwidths, alone, were too narrow for the detection of phase shifts.
\end{abstract}

\section{Results and Discussion}

Employing the identical measurement procedures as before, Figure 4 shows phase-shift detection scores [P(A)] as a function of phase angle and spectral distance between the low reference (correlated) and test (phase-shifted) frequency bands. These data further confirm the importance of spectrally adjacent frequency information for the detection of phase shifts, as evidenced by the marked drop in sensitivity from the frequency band $4<\mathrm{f} \leq 8 \mathrm{cpd}$ to the others.

One prediction from the above is that the detection of phase shifts flanked (on both sides) by correlated spectral components would result in improved sensitivity to phase shifts (relative to the absence of such flanking components) for any spectral region. This prediction, and the 


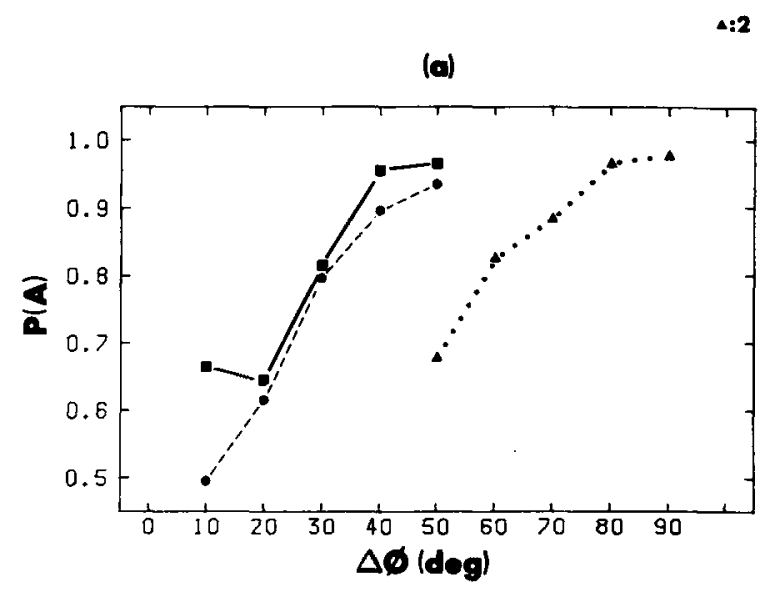

๑.3 $0: 4$

Мн

(b)

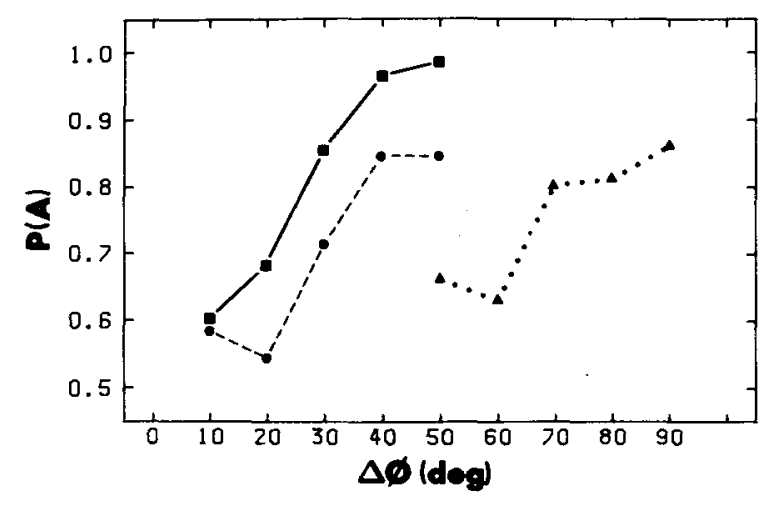

(c)

WE

(d)
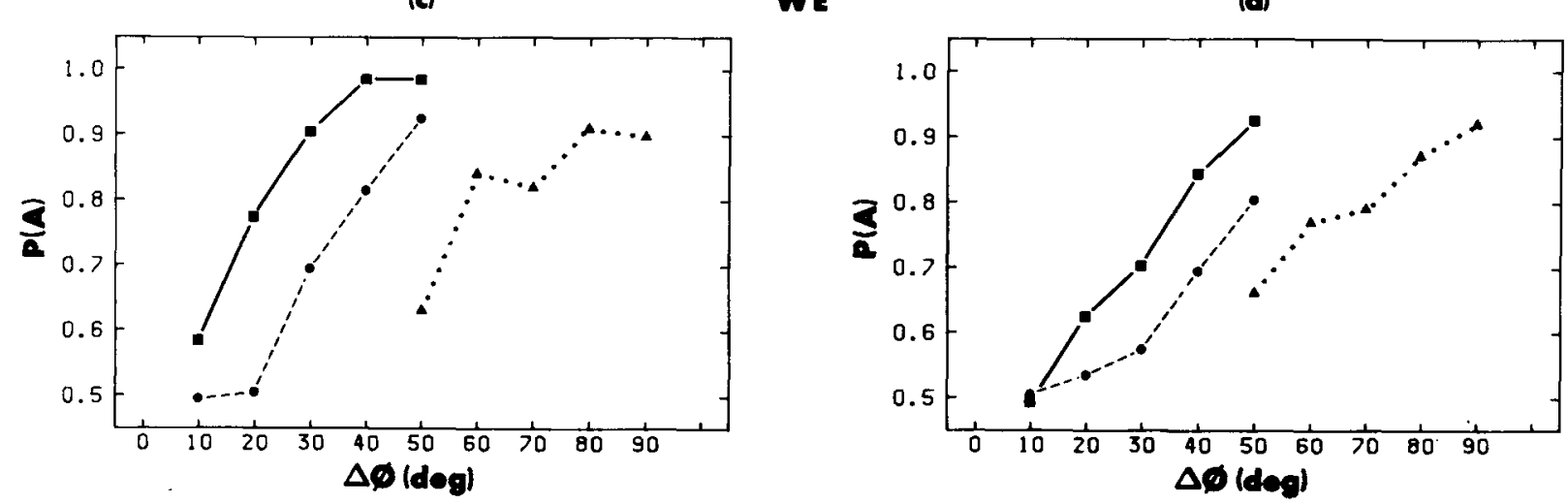

Figure 3. Phase shift $(\Delta \phi$ in degrees) detection $[\mathrm{P}(\mathrm{A})]$ as a function of frequency bandwidth (of $\Delta 2-, \bullet 3-$, and $\square 4-0 c t a v e$ widths) at two center frequencies $[(a)+(c): 6$ and $(b)+(d): 12$ cpd] for two observers (M.H. and W.E.).

results of Experiment 2 run counter to what would be expected from a masking perspective, since, in masking experiments, sensitivity decreases as frequencies converge. The aim of the final experiment was to examine this prediction in some detail.

\section{EXPERIMENT 3:}

\section{Sensitivity to Phase Shifts in Correlated Bands}

In this experiment, we employed four 1-octave-wide (picture cycle) annuli defined by $\mathrm{B}_{1}: 2<\mathrm{f} \leq 4, \mathrm{~B}_{2}$ : $3<\mathrm{f} \leq 6, \mathrm{~B}_{3}: 4<\mathrm{f} \leq 8, \mathrm{~B}_{4}: 6<\mathrm{f} \leq 12 \mathrm{cpd}$, and a fifth $\left(B_{5}\right)$ total spectrum. As already demonstrated in Experiments 1 and 2, phase shifts within these bands are not detectable when presented alone or within spectrally distant information. However, here we have not altered any amplitude components and have introduced phase shifts only within each specified band. Examples are shown in Figure 5.

\section{Method}

The procedures were identical to those used in the previous two experiments, with each observer being presented with $140 \times 5$ (bands) $\times 3$ (repeats) $=2,100$ trials.

\section{Results}

Figure 6 shows the results for the two observers over the five phase-shift regions. These curves were fitted by logistic regression over combined subject responses to result in $75 \%$ detection phase-shift values of (a) $12^{\circ}$ for $4<\mathrm{f} \leq 8 \mathrm{cpd}$, (b) $20^{\circ}$ for $6<\mathrm{f} \leq 12 \mathrm{cpd}$, (c) $21^{\circ}$ for $8<\mathrm{f} \leq 16 \mathrm{cpd}$, (d) $27^{\circ}$ for $1<\mathrm{f} \leq 24 \mathrm{cpd}$, and (e) $19^{\circ}$ for the complete spectrum $(0<\mathrm{f} \leq 32 \mathrm{cpd})$. This result replicates Caelli and Bevan's (1982) previous finding that sensitivity decreased as the 1-octave-wide phase-shifted region increased in center frequency [(a)(c) above, see Figure 6]. However, we note that this decrease occurs in regions in which both spatial-frequency sensitivity and signal energy are reduced. Again, we find here that the sensitivity to shifts of the entire spectrum is no better than that for the best frequency band. This lack of improved sensitivity to total-spectrum phase shifts is also parsimonious with the conclusion that sensitivity to phase shifts is accomplished by the perceptual correlation of spectrally proximal spatial information (that is, the correlation of similar luminance gradients). In the case where narrow-band phase-shifted signals were embedded in spatial information that is perfectly correlated, we would expect performance to be slightly improved over 
(a) $\mathrm{MH}$

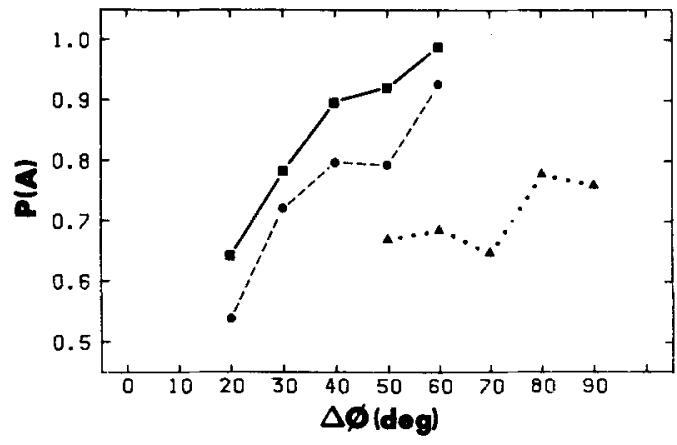

(b) WE

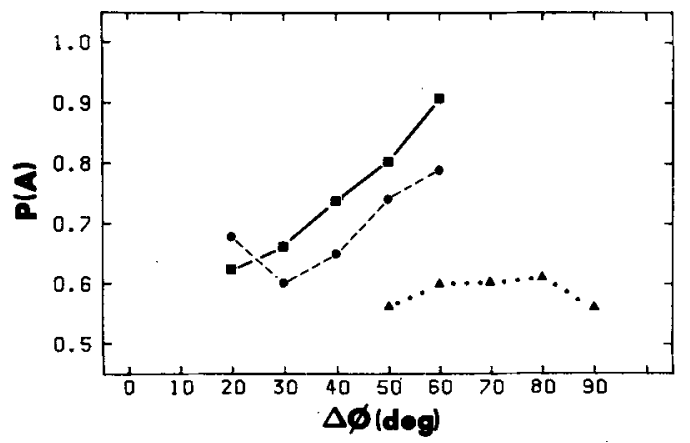

Figure 4. Phase shift ( $\Delta \phi$ in degrees) detection $[\mathrm{P}(\mathrm{A})]$ as a function of the spectral separation between a low-frequency correlated band $(0<\mathrm{f} \leq 4 \mathrm{cpd})$ and phase-shifted (1-octave) frequency bands of $04<f \leq 8, * 6<f \leq 12$, and $\Delta 8<f \leq 16 \mathrm{cpd}$, for two observers (M.H. and W.E.).

that found with shifts induced in the complete spectrum. In this latter case, the neighboring reference bandwidths also have decorrelated information, thus making the task more difficult. This increased difficulty is compensated for by an increase in the energy and bandwidth of the decorrelating shifting window. Consequently, we would expect this full-spectrum-shift case to be of moderate difficulty.

The corresponding decorrelation (or shifting) windows for each of the four frequency bands at $30^{\circ}$ phase shifts have lower and upper (annuli) radii (r) of: by $B_{1}$ : $1.25^{\prime} \geq r \geq 0.75^{\prime}, \quad B_{2}: 0.84^{\prime} \geq r \geq 0.42^{\prime}, \quad B_{3}$ : $0.75^{\prime} \geq r \geq 0.38^{\prime}, \quad B_{4}: 0.42^{\prime} \geq r \geq 0.21^{\prime}$. Consequently, the results suggest that the detection of decorrelations between images is accomplished via a hierarchy of different-sized gradient registration detectors whose acuities increase with a signal's center frequency.

\section{GENERAL DISCUSSION}

In previous experiments (Caelli \& Bevan, 1982), it has been demonstrated that human observers cannot detect, or perceptually resolve, phase angles smaller than $15^{\circ}$ (often considerably larger than this) in any twodimensional spectral region of suprathreshold images. The present experiments have confirmed these limits, this time with a different phase modulation operation, and have extended the findings to include bandwidth and reference frequency effects.

Our results can be summarized as follows: (1) For isolated band-limited signals, to a maximum of 1 octave, phase shifts are not detectable. However, in the presence of other broadband image components, such 1-octavewide phase shifts are equally detectable over low-, middle-, and high-frequency ranges. That is, such additional image information apparently serves as a reference frame, thereby facilitating the detection of phase shifts. (2) As the frequency bandwidth of a signal increases upwards from 1 octave, sensitivity improves and apparently reaches a ceiling at about a 3-octave width (Experiment 1), independent of the center frequency. (3) Sensitivity for 1octave-wide phase shifts decreases with increases in the spectral distance between the band and a fixed reference (correlated) frequency band (Experiment 2).

These results strongly suggest that the detection of modulations in phase, from a frequency domain perspective, is not accomplished by the correlation of the responses of widely separated channels. Rather, our data indicate that phase sensitivity comes about via the correlation of spectrally proximate spatial-luminance profiles (that is, similar luminance gradients). The nature of this correlation process is the subject of a recent study by Caelli and Rentschler (1985).

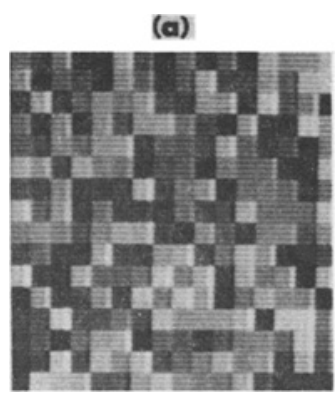

(b)

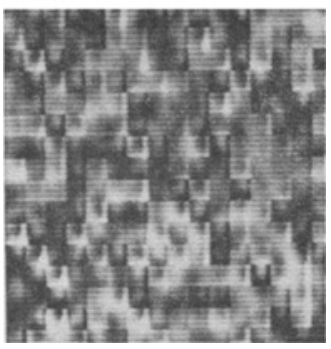

(c)

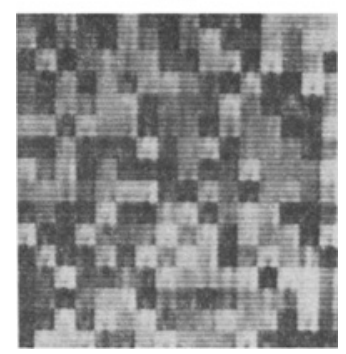

Figure 5. (a) Original $128 \times 128$ 6-bit pixel texture (b and c). Examples of phase shifts of $50^{\circ}\left(\Delta \phi=50^{\circ}\right)$ in low [(b): $\left.4<f \leq 8 \mathrm{cpd}\right]$ and high [(c): $8<\mathrm{f} \leq 16 \mathrm{cpd}]$ frequency bands. Here amplitude (and phase) components outside each region are preserved. 


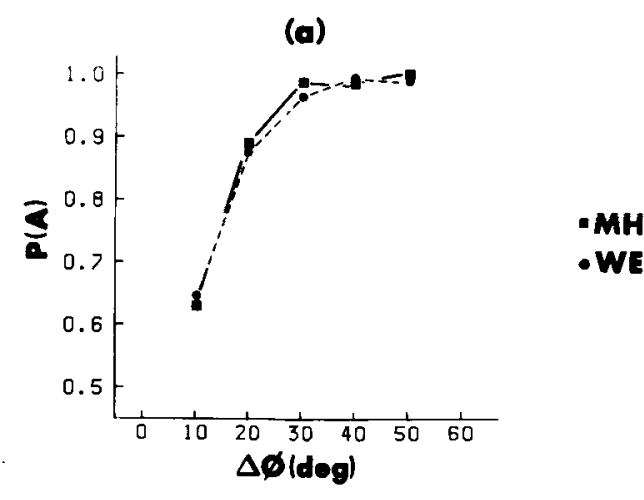

(b)

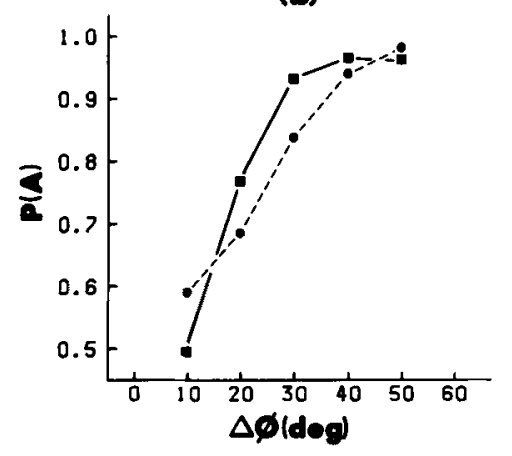

(d)

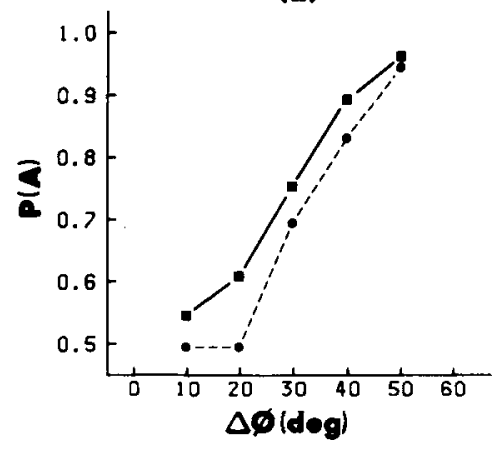

(c)

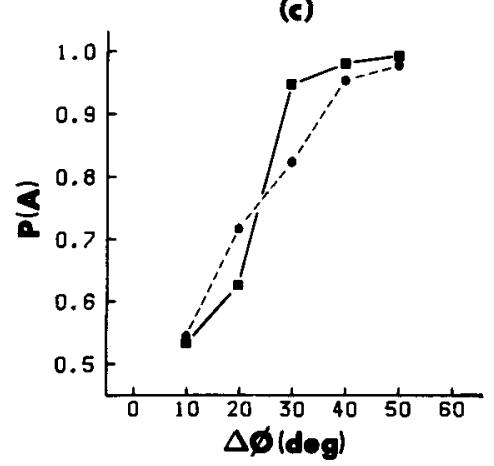

(o)

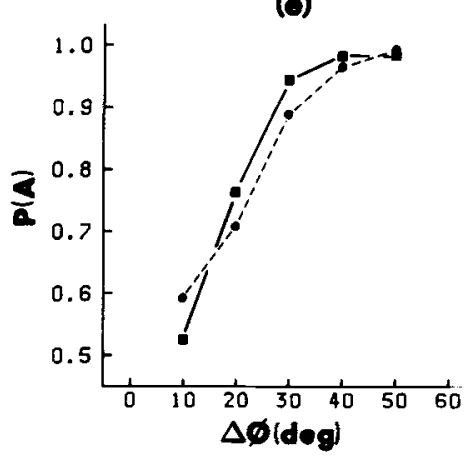

Figure 6. Phase-shift ( $\Delta \phi$ in degrees) detection [P(A)-area under the ROC curve] as a function of the frequency range of the 1-octave-wide bands specified in picture cycles: (a) $4<$ f $\leq 8$, (b) $6<$ f $\leq 12$, (c) $8<$ f $\leq 16$, (d) $1<$ f $\leq 24$, and (e) complete spectrum: $0<\mathrm{f} \leq 32$ cpd.

Most other investigations of phase sensitivity have been restricted to one-dimensional threshold grating studies (e.g., Burr, 1979). The results of those studies have limited application to suprathreshold two-dimensional image processing for two reasons. First, phase modulations of one-dimensional grating patterns induce specific patterns of luminance modulations that are difficult to generalize to arbitrary shapes or luminance profiles. Second, phase encoding is specifically sensitive to orientation components in the image. For example, if the "reference" grating were perpendicular to the direction of phase shift, phase detection would virtually disappear, since the luminance profiles remain unaltered. It is for these reasons that the geometry of the two-dimensional phase-shifting window has to be considered before conclusions can be made about phase encoding in visual image processing.

For completeness, it should be noted that the operation of phase shifting is analagous to what is known as birefringence in physical optics. Indeed, a narrow beam of light incident to an anisotropic crystal will split and emerge as two parallel beams. Thus, double images are formed by such crystals. This phenomenon depends upon the decomposition of plane electromagnetic waves into components traveling with different phase velocities, as determined by the anisotropy in the refractive index (Born \& Wolf, 1970, pp. 684-690). The spatial displacement 
between the two components results from the difference in refraction. In the context of the present study, images obtained by introducing a spatial shift would correspond to the generation of "extraordinary" waves. Similar to the processes of holography, our data demonstrate the importance of a reference frequency band in the detection of phase shifts. However, we propose that, unlike holography, this reference corresponds to a relatively wide, but spectrally proximate, signal and the mechanism responsible for determining phase sensitivity is a complex crosscorrelation process.

\section{REFERENCES}

Atkinson, J., \& Campbell, F. W. (1974). The effect of phase on the perception of compound gratings. Vision Research, 14, 159-162.

Born, H., \& Wolf, E. (1970). Principles of optics (4th ed.). Oxford: Pergamon Press.

Brettel, H., Caelli, T., Hilz, R., \& Rentschler, I. (1982). Modelling perceptual distortion: Amplitude and phase transmission in the human visual system. Human Neurobiology, 1, 61-67.

BURR, D. (1979). Sensitivity to spatial phase. Vision Research, 20, 391-396.

Burton, G. J., \& Moorhead, I. R. (1981). Visual form perception and the spatial phase transfer function. Journal of the Optical Society of America, 71, 1056-1063.

CAELLI, T. (1981). Visual perception: Theory and practice. Oxford: Pergamon Press.

Caell, T., \& Bevan, P. (1982). Visual sensitivity to two-dimensional spatial phase. Journal of the Optical Society of America, 72, 1375-1381.

Caelli, T., \& Hübner, M. (1983). On the efficient two-dimensional energy coding characteristics of spatial vision. Vision Research, 23, 1053-1055.
CAELLI, T., \& HüBnER, M. (1984). On the number of intensity levels detected in textures. Perception, 13, 21-31.

CAELli, T., \& Rentschler, I. (1985). A cross-correlation model for pattern acuity. Manuscript submitted for publication.

Dorfman, D. D., \& AlF, E., JR. (1969). Maximum likelihood estimation of parameters of signal-detection theory and determination of confidence intervals-rating method data. Journal of Mathematical Psychology, 6, 487-496.

Gayl, I. E., Strasburger, H., Rentschler, I., \& Hilz, R. (1982). Evoked potentials and psychophysics of spatial phase sensitivity. Journal of the Optical Society of America, 72, 68.

Green, D., \& SWETS, J. (1974). Signal detection theory and psychophysics. New York: Krieger.

HARVEY, L. O., JR. (1984), Estimation of the reliability of signal detection parameters Unpublished manuscript. (University of Munich)

NaChmias, J., \& WEBER, A. (1975). Discrimination of simple and complex gratings. Vision Research, 15, 217-223.

OPPENHEIM, A., \& LIM, P. (1981). The importance of phase in signals. Proceedings, IEEE, 69, 529-541.

WeSTHEIMER, G. (1978). Spatial phase sensitivity for sinusoidal grating targets. Vision Research, 18, 1073-1074.

\section{NOTE}

1. To preserve real (inverse) images, the phase shifts for the discrete Fourier transform algorithm must take the form of $\Delta \phi$ for $(u, v)$, where $v>0$, and $-\Delta \phi$ for $v<0$. This guarantees that in the phase-shifted $\left(\phi^{\prime}\right)$ spectrum:

$$
\phi^{\prime}(\mathrm{u}, \mathrm{v})=-\phi^{\prime}(-\mathrm{u},-\mathrm{v})
$$

(Manuscript received August 24, 1984; revision accepted for publication May $7,1985$. ) 